\title{
ARTICLE
}

Received 16 Feb 2016 | Accepted 25 Oct 2016 | Published 23 Dec 2016

DOI: $10.1038 /$ ncomms 13769

\section{Single-cycle surface plasmon polaritons on a bare metal wire excited by relativistic electrons}

W.P.E.M. op 't Root', G.J.H. Brussaard', P.W. Smorenburg' \& O.J. Luiten ${ }^{1,2}$

Terahertz $(\mathrm{THz})$ pulses are applied in areas as diverse as materials science, communication and biosensing. Techniques for subwavelength concentration of $\mathrm{THz}$ pulses give access to a rapidly growing range of spatial scales and field intensities. Here we experimentally demonstrate a method to generate intense $\mathrm{THz}$ pulses on a metal wire, thereby introducing the possibility of wave-guiding and focussing of the full $\mathrm{THz}$ pulse energy to subwavelength spotsizes. This enables endoscopic sensing, single-shot subwavelength $\mathrm{THz}$ imaging and study of strongly nonlinear $\mathrm{THz}$ phenomena. We generate $\mathrm{THz}$ surface plasmon polaritons (SPPs) by launching electron bunches onto the tip of a bare metal wire. Bunches with $160 \mathrm{pC}$ charge and $\approx 6 \mathrm{ps}$ duration yield SPPs with $6-10 \mathrm{ps}$ duration and $0.4 \pm 0.1 \mathrm{MVm}^{-1}$ electric field strength on a $1.5 \mathrm{~mm}$ diameter aluminium wire. These are the most intense SPPs reported on a wire. The SPPs are shown to propagate around a $90^{\circ}$ bend.

\footnotetext{
${ }^{1}$ Coherence and Quantum Technology, Department of Physics, Eindhoven University of Technology, Eindhoven 5600 MB, The Netherlands. ${ }^{2}$ Institute for Complex Molecular Systems, Eindhoven University of Technology, Eindhoven 5600 MB, The Netherlands. Correspondence and requests for materials should be addressed to O.J.L. (email: o.j.luiten@tue.nl).
} 
T he terahertz $(\mathrm{THz})$ part of the electromagnetic spectrum $(0.1-10 \mathrm{THz})$ is ideal for probing and manipulating fundamental excitations in solids and rotational states in molecules. The development of $\mathrm{THz}$ techniques in recent years has led to many exciting applications, such as medical imaging, characterization of materials, security screening and industrial process control. In particular, the development of high-intensity, pulsed $\mathrm{THz}$ sources based on rectification of femtosecond laser pulses has revolutionized $\mathrm{THz}$ science and technology ${ }^{1-5}$. Even more spectacular progress in this vibrant field is expected from the development of $\mathrm{THz}$ plasmonics, presently a very active area of research, enabling new ways to control and manipulate $\mathrm{THz}$ radiation ${ }^{6,7}$.

By providing guided delivery of intense $\mathrm{THz}$ pulses, $\mathrm{THz}$ waveguides ${ }^{8}$ open up new opportunities in photonics, such as single-shot, subwavelength $\mathrm{THz}$ imaging and possibly new applications based on nonlinear $\mathrm{THz}$ phenomena ${ }^{9}$. In cancer research, endoscopic systems based on wave-guiding intense $\mathrm{THz}$ pulses could greatly extend $\mathrm{THz}$ cancer detection schemes, which are today limited to tissue that can be reached with optical techniques ${ }^{10,11}$. By tapering a THz wire waveguide into a tip, the $\mathrm{THz}$ pulses can be focussed to subwavelength spot-sizes, which further increases the intensity ${ }^{12-20}$. One interesting application of this is a new class of pulsed electron guns that use the wire tip as an electron emitter with record brightness ${ }^{21}$. Moreover, the subwavelength focussing of $\mathrm{THz}$ pulses provided by tapered wire waveguides greatly improves the spatial resolution of $\mathrm{THz}$ experiments ${ }^{12-20}$. In recent years, several methods have been devised to localize free-space $\mathrm{THz}$ radiation to nanometre-sized structures such as atomic force microscopic tips ${ }^{22}$ and nanolithography-based slits ${ }^{23}$, in which a small fraction of the $\mathrm{THz}$ pulse is locally enhanced to the $\mathrm{kV} \mathrm{cm}^{-1}$ range at the sample with spatial resolutions in the nanometre range. Even subcycle temporal resolution of $10 \mathrm{fs}$ has been achieved based on gated, multi-shot measurements using multicycle $\mathrm{THz}$ pulses ${ }^{22}$. This fascinating field can be further developed by using strong subcycle $\mathrm{THz}$ pulses that are wire-guided, which enables focussing of the full $\mathrm{THz}$ pulse energy to subwavelength distances, while providing temporal resolution through the subcycle $\mathrm{THz}$ pulse length. This will enable single-shot subwavelength $\mathrm{THz}$ imaging and the study of strongly nonlinear THz phenomena'.

Accelerator-based systems and optical techniques exist to produce short intense $\mathrm{THz}$ pulses propagating in free space ${ }^{1-5,24-26}$. Such $\mathrm{THz}$ pulses have been coupled onto wire waveguides where they propagate as surface plasmon polaritons (SPPs) along the surface of the wire ${ }^{8,27-32}$. Unfortunately, creating SPPs by coupling free-space $\mathrm{THz}$ radiation onto a metal wire is inefficient owing to the very poor spatial overlap between the linearly polarized free-space mode and the radially polarized waveguide mode $28,29,31,32$. Additionally, direct SPP excitation techniques involving electron beams interacting with flat surfaces have been demonstrated ${ }^{33}$ but not on a wire. In 2008, we proposed a method in which ultrashort bunches of relativistic electrons generate coherent transition radiation (CTR) at the tip of a thin wire ${ }^{34}$. The radiation produced then propagates as a powerful SPP along the wire, which serves as a waveguide. This hybrid technique integrates accelerator-based CTR methods and plasmonic techniques to produce intense subcycle pulses in the $\mathrm{THz}$ frequency range directly on a bare metal wire waveguide ${ }^{34}$. By this method, extremely intense and highly localized $\mathrm{THz}$ pulses can be created using state-of-the-art table-top-sized accelerators ${ }^{35}$. The $\mathrm{GeV}$ electron bunches currently driving $\mathrm{X}$-ray free electron lasers (X-FELs) ${ }^{25}$ would enable nonlinear 'THz pump-X-ray probe' experiments in which micron-sized pieces of material are excited by extreme $\mathrm{THz}$ field strengths and subsequently probed by X-ray pulses to study structural dynamics at femtosecond timescales.

The proposed method relies on the natural match of the Coulomb field of relativistic electron bunches with the radially polarized electric field of the guided surface wave. Firing electron bunches onto the tip of a tapered metal wire causes the radially polarized field of the electron bunches to transfer to the wire. This results in SPPs propagating along the wire. The physical mechanism behind this process can be understood using a field line model as described by Purcell ${ }^{36}$, which is described in the Methods section. From this model, the estimated peak electric field strength $E$ of the SPP generated on the wire is given by

$$
E=C_{\mathrm{s}} q / 2 \pi \varepsilon_{0} c \tau R
$$

where $q$ is the electron bunch charge, $\tau$ the bunch duration, $R$ the radius of the wire, $\varepsilon_{0}$ the vacuum permittivity and $c$ the speed of light. The numerical dimensionless constant $C_{\mathrm{s}}$ takes the threedimensional bunch shape into account and depends mildly on the beam energy; $C_{\mathrm{s}}$ can be calculated using more elaborate methods ${ }^{34}$. For ellipsoidal bunches with a length $c \tau$ (semiaxis) and $2 \mathrm{MeV}$ energy, $C_{\mathrm{s}} \approx 0.7$. Meanwhile, the length of the SPP is comparable to that of the generating electron bunch, with a spectrum coherent up to frequencies $\sim 1 / \tau$.

Here we experimentally demonstrate the method proposed in ref. 34. By firing electron bunches with an energy of $3.1 \mathrm{MeV}$, bunch charge $q=160 \mathrm{pC}$ and full-width-at-half-maximum (FWHM) duration of $\tau \approx 6 \mathrm{ps}$ on a sharp conical tip of an aluminium wire with a diameter of $1.5 \mathrm{~mm}$, we generate SPPs with a FWHM duration of 6-10 ps and electric field strength of $0.4 \pm 0.1 \mathrm{MV} \mathrm{m}^{-1}$ on the surface of the wire. This yields the possibility of waveguiding and subwavelength focussing of the full $\mathrm{THz}$ pulse, enabling endoscopic delivery and study of strongly nonlinear $\mathrm{THz}$ phenomena. Equation (1) shows that the SPP field strength on the wire is proportional to the peak electron beam current $\sim q / \tau$. Using the $100 \mathrm{fs}, 100 \mathrm{pC}, 3.7 \mathrm{MeV}$ bunches that can be produced in a table-top radio frequency photogun setup comparable to ours ${ }^{35}$, $\mathrm{THz}$ field strengths of tens of $\mathrm{MV} \mathrm{m}^{-1}$ could be generated on a millimetre-diameter wire. A fortiori, the sub-100-fs, nanocoulomb electron bunches driving X-FELs have the potential to generate $\mathrm{THz}$ pulses on a millimetre-diameter wire in excess of $1 \mathrm{GVm}^{-1}$, comparable to the record field strengths obtained by free-space CTR at Stanford Linear Accelerator Center (SLAC) ${ }^{25}$.

\section{Results}

Experimental setup. The basic principle of using an ultrashort bunch of relativistic electrons to produce CTR at the tip of a thin wire, which subsequently propagates along the wire as a powerful SPP, is illustrated in Fig. 1 and is explained in more detail in the Methods section. We have demonstrated the method in a smallscale experimental setup, which is schematically illustrated in Fig. 2. An animation of the experiment (Supplementary Movie 1) can be found in Supplementary Information. Electron bunches from a $3.1 \mathrm{MeV}$ accelerator containing $160 \mathrm{pC}$ of charge are focussed onto the conical tip of an aluminium wire. The radius $R$ of the wire is $0.75 \mathrm{~mm}$ and the conical tip has an opening angle $2 \delta=8^{\circ}$. At the tip of the wire, the electron bunches have a radius of $0.2 \mathrm{~mm}$ (root mean square (RMS)). When the electrons hit the wire, they are absorbed into the wire, setting up a pulse of transition radiation that propagates along the surface of the wire as an SPP. This surface wave is analysed at the end of the straight part (1) of the wire (see Fig. 2), $80 \mathrm{~mm}$ from the tip, by placing a $\mathrm{ZnTe}<110>$ crystal $\left(5 \times 5 \times 0.5 \mathrm{~mm}^{3}\right)$ directly on the surface of the wire. A circularly polarized pulse $(50 \mathrm{fs}, \sim 1 \mu \mathrm{J}, 800 \mathrm{~nm})$ from the Ti:Sapphire laser system is used to probe the crystal. These probe pulses come from the same laser system that is used to generate the electrons through photo-emission in the accelerator 
a

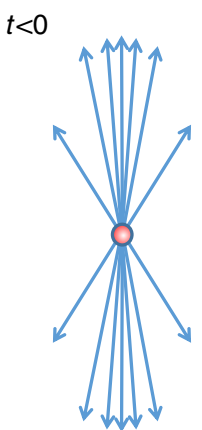

$t<0$

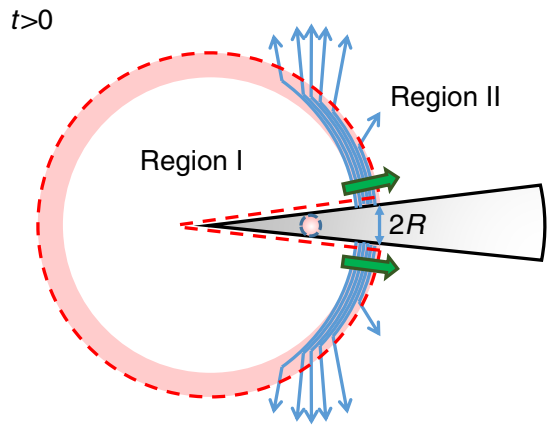

Figure 1 | Field line model of SPP formation by firing an electron bunch onto a wire. (a) Electric field lines of the electron bunch before impact. (b) Field lines shortly after impact. The dashed red line indicates the sphere with radius $c \tau$ and conical cut-out (that is, the Gauss volume used in the text). Inside region I, the electric field of the electron bunch is completely screened by the metal wire. The field in region II is that of an undisturbed electron. At the boundary between the two regions, a thin shell of dense field lines is formed (shaded band). The thick green arrows indicate the concentration of field lines forming an SPP that propagates onto the wire.

and are therefore synchronous with the electron bunches and the SPP. The ZnTe crystal is imaged through a polarizer cube onto two charge-coupled device (CCD) cameras. By taking the relative difference of the two images $[(A-B) /(A+B)]$, an image of the field strength of the surface wave in the ZnTe crystal is obtained. Just behind the $\mathrm{ZnTe}$ crystal, the wire forms a bend, with a radius of curvature of $33 \mathrm{~mm}$. After the bent section (2) of the wire, a second $\mathrm{ZnTe}$ crystal is placed on the wire to determine the field strength of the SPP at this position. Because the electric field of the SPP is weaker after the bend, we do not image this crystal but instead use a balanced diode setup to record the change in polarization of the probe pulses induced by the SPP in the ZnTe crystal. By varying the path length of the probe pulses with respect to the photo-emission pulses, a time scan of the signal is formed $^{37-40}$.

SPP measurements before the bend. Fig. 3a shows the image of the first ZnTe crystal, illuminated by the probe pulse at the moment the SPP passes the crystal. The probe pulse grazes the wire to allow imaging of the point at which the crystal touches the wire. As the probe pulse comes in at a slight angle in the $x$ direction (see Fig. 2) and the $y$ direction (not shown in Fig. 2), this causes the wire to shade off the lower left corner of the crystal. The relative difference between the intensities reaching both cameras is proportional to the vertical component of the electric field of the SPP inside the ZnTe crystal. The procedure that we used to calculate the absolute value of the electric field and colour code the image of Fig. 3a is described in the Methods section. The image was taken at the maximum field strength of the SPP. The line-out along the vertical axis at $x=0$ is plotted in Fig. 3b. For a cylindrically symmetric SPP on a metal wire with radius much larger than the skin-depth, one expects the radial component of the electric field to fall off as $r^{-1}$ (ref. 36,41), with $r$ the radial distance from the wire centre. However, the $\mathrm{ZnTe}$ crystal, with a dielectric constant of approximately 10 for the relevant frequencies, artificially enhances the field near the wire surface owing to refraction. We modelled this effect using CST Microwave Studio. At $r \geq 2 R$, the electric field shows the expected $r^{-1}$ dependence in both the measurement of Fig. 3b and the CST a

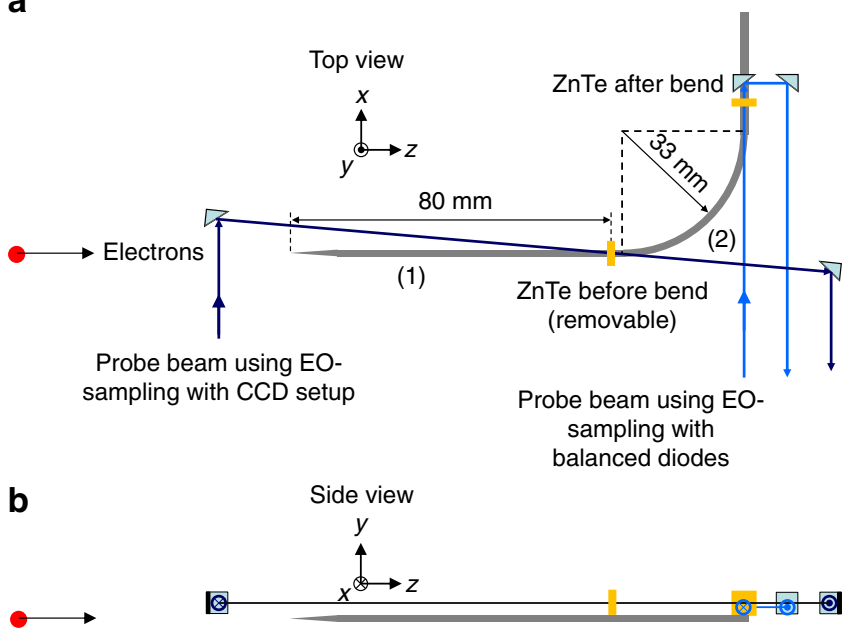

Figure 2 | Experimental layout. (a) Top view of the used experimental layout. (b) Side view of the used experimental layout. When an electron bunch hits the tip of the wire, part of the bunch is absorbed, generating a surface wave propagating along the wire. When the surface wave passes the first ZnTe crystal at the end of the straight section (1), it induces birefringence in the crystal that changes the polarization of the probing laser pulse. After reflection on a turning mirror, the laser pulse exits the chamber and is imaged onto two CCD cameras (not shown here) to obtain the image shown in Fig. 3a. To measure the field strength of the surface wave after propagating around the bent section (2) in the wire, the first ZnTe crystal (before the wire bend) is removed. In this configuration, the probing laser pulses pass through the second ZnTe crystal and are analysed using two photodiodes to measure the change in polarization. By varying the delay between the electron bunches and the laser pulses, the time dependence of the electric field of the surface wave is obtained, shown in Fig. 4. The coordinate system shown in this figure is used throughout the paper.

simulation. To extract the undistorted field from the measurements, we therefore fit the data between $y=1.25 \mathrm{~mm}$ and $2.75 \mathrm{~mm}$ with this $r^{-1}$ dependence and extrapolate to the wire's edge at $y=0.75 \mathrm{~mm}$ (dashed line in Fig. 3b). Finally, we correct for the transmission coefficient of the crystal $\left(t_{\mathrm{ZnTe}}=0.48\right.$ at $1 \mathrm{THz})$ to calculate what the electric field of the SPP would have been without the crystal present. This is shown as the dotted line in Fig. 3b. We thus find that SPPs have been generated directly on a wire by relativistic electron bunches and that the maximum field strength of the SPPs was $0.4 \pm 0.1 \mathrm{MV} \mathrm{m}^{-1}$.

By changing the delay between the photo-emission laser pulse and the probe pulse through the $\mathrm{ZnTe}$ crystal, we can now determine the field strength on the wire surface as a function of time. The result of such a scan is shown in Fig. 4a. The RMS length of the pulse is between 2 and 3 ps, depending on the level of the baseline that has an uncertainty of $\pm 0.1 \mathrm{MV} \mathrm{m}^{-1}$. The signal does not quite return to zero for short delay times, which may be a result of drift in the calibration of the electro-optical system. We are therefore cautious to put a more precise number on the measured pulse duration.

SPP measurements after the bend. To demonstrate that the SPP is guided by the wire, we have also measured the electric field strength after the $90^{\circ}$ bend in the wire using the second $\mathrm{ZnTe}$ crystal (see Fig. 2). The laser probe pulses were focussed on the crystal with a spot size of $120 \pm 20 \mu \mathrm{m}$ (FWHM), at a distance of $1.0 \pm 0.8 \mathrm{~mm}$ from the edge of the wire. During these measurements, the crystal before the bend was removed to avoid disturbing the SPP. Using the same $r^{-1}$ scaling as in the imaging 

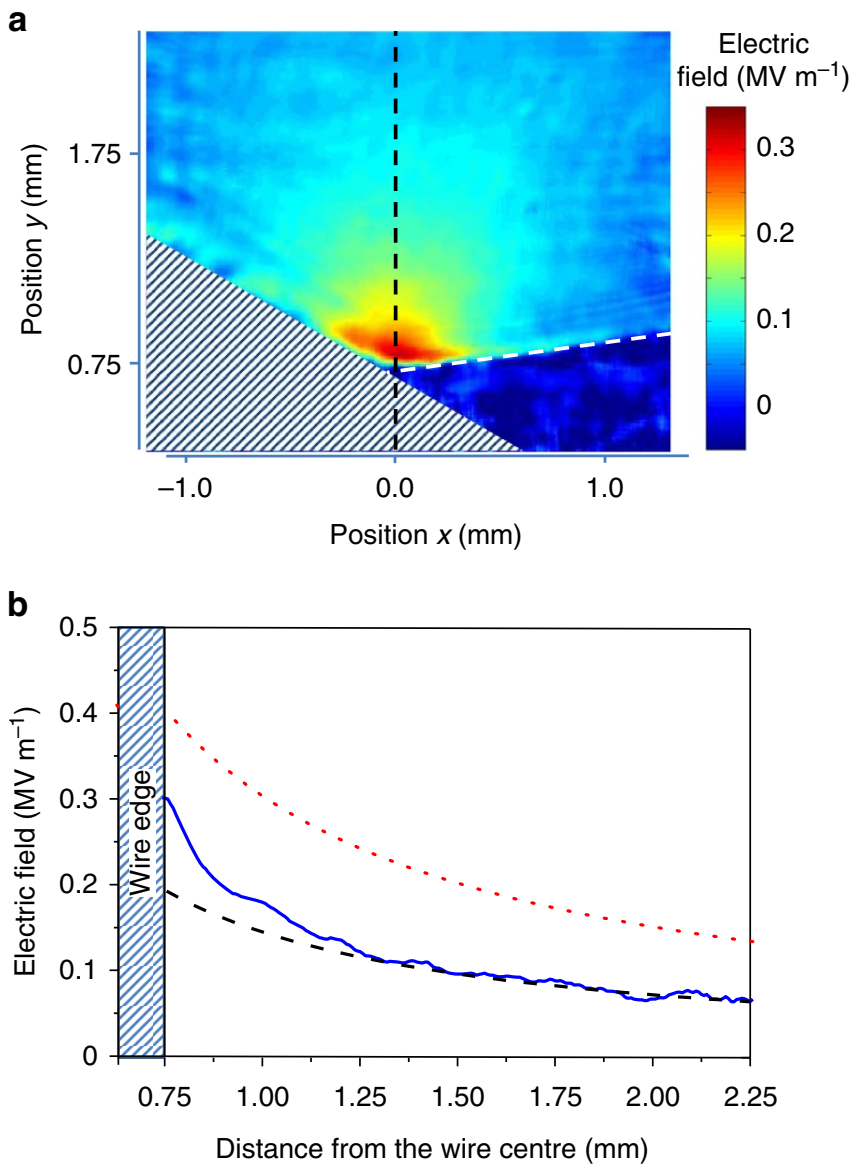

Figure 3 | Field strength of the SPP at $80 \mathrm{~mm}$ from the tip of the wire. (a) Image of the ZnTe crystal touching the wire surface. The colour code represents the relative intensity of the laser pulse passing through the crystal and an analyser and is a measure of the change in polarization caused by the birefringence induced in the crystal by the $y$-component of the electric field of the SPP. The hatched region is the shadow of the wire; the dashed line indicates the edge of the crystal. (b) Line-out along the vertical axis $x=0$ of (a) (blue solid line). The black dashed line shows the expected $r^{-1}$ dependence of the electric field strength at distances greater than $y=1.25 \mathrm{~mm}(0.5 \mathrm{~mm}$ from the surface of the wire). This line is extrapolated to show the field enhancement near the surface of the wire caused by the mismatch between the permittivity of the crystal and vacuum. The red dotted line is the calculated field strength of the SPP in the absence of the crystal.

technique above, we can calculate the undisturbed field on the surface of the wire in order to compare it with the field strength of the SPP before the bend. The results are shown in Fig. 4. Owing to the uncertainty in the exact position of the laser spot with respect to the edge of the wire, the absolute values of the field strength in Fig. 4 may be between $30 \%$ lower and $75 \%$ higher, corresponding to an amplitude transmission coefficient of $20-50 \%$.

\section{Discussion}

We performed particle tracking simulations (General Particle Tracer ${ }^{42}$ ) to calculate the expected duration of the electron bunches at the moment they enter the tip of the wire. These simulations predict an RMS bunch length of $2.7 \mathrm{ps}$ for bunches of $160 \mathrm{pC}$ in the present setup. This is in reasonable agreement with the observed RMS pulse duration of the SPP of 2-3 ps. With Equation (1), we can now calculate the expected maximum a

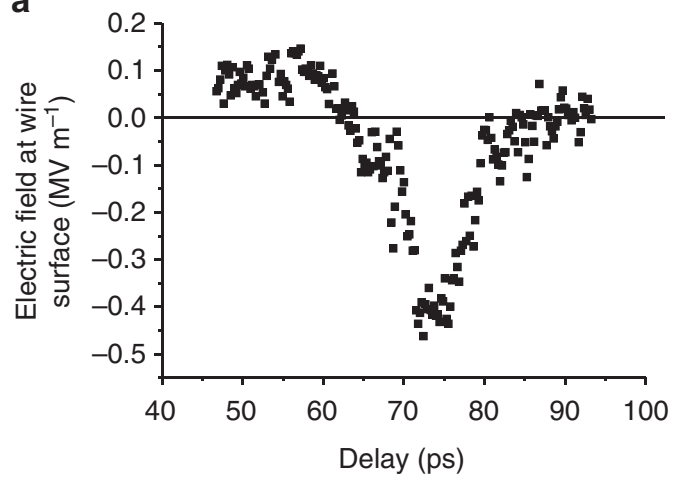

b

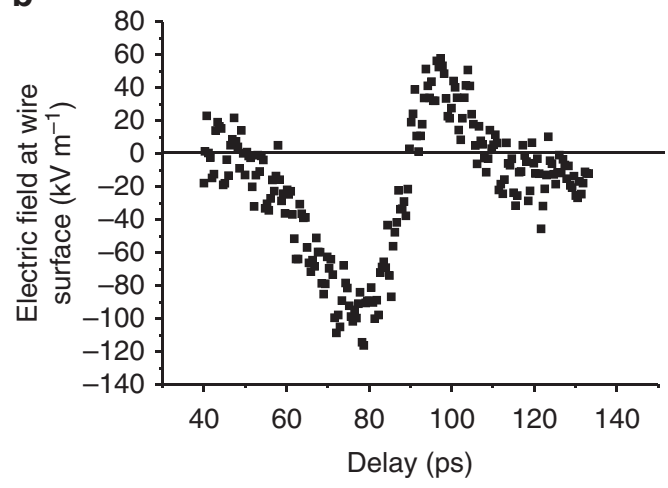

Figure 4 | Electric field of the SPP at the surface of the wire as a function of time. (a) Measurements of the field strength on the wire surface taken at the position $z=80 \mathrm{~mm}$ from the tip of the wire. For each setting of the time delay between the probe pulse and the laser pulse used for photoemission of the electron bunch, the electric field strength at the surface of the wire was extracted from images recorded in the same manner as Fig. 3. (b) Field strengths measured after the bend in the wire $(33 \mathrm{~mm}$ radius of curvature) using a balanced diode set-up instead of the double-CCD camera used in (a). Zero delay is arbitrary for both $(\mathbf{a}, \mathbf{b})$.

electric field of the SPP. Taking an equivalent ellipsoidal electron bunch with the same RMS bunch length, this gives a peak electric field of $1.7 \mathrm{MV} \mathrm{m}^{-1}$. The measured field strength on the surface of the wire, corrected for the presence of the $\mathrm{ZnTe}$ crystal is $0.4 \mathrm{MV} \mathrm{m}^{-1}$ (Fig. 3b), that is, approximately a factor 4 lower than predicted for the experimental conditions used here. A possible explanation may be found if we consider the fact that the range of $3 \mathrm{MeV}$ electrons in aluminium is $6 \mathrm{~mm}$. The electrons therefore penetrate only the initial part of the conical tip and a significant part of the bunch is scattered into large angles, that is, sideways out of the wire. These electrons do not contribute to the CTR pulse. Furthermore, electrons that are absorbed by the wire may eject secondary electrons, which partly cancel the transition radiation field of the absorbed electrons. Nevertheless, the measured field strength is the highest reported to date measured at the surface of a metal wire. Comparison of these results to other techniques that generate SPPs directly on a wire is difficult, as the field strength is often reported in arbitrary units. In fact, we could not find any article reporting the electric field strength of SPPs on a metal wire surface.

By propagating around a $90^{\circ}$ bend with $33 \mathrm{~mm}$ radius of curvature, the FWHM SPP pulse length increases from 6-10 ps to approximately $16 \mathrm{ps}$, while the maximum SPP electric field strength is reduced to $20-50 \%$ of its original value. On the basis of the measurements reported by Astley et al. ${ }^{43}$, we expected an 
amplitude transmission of around $15 \%$ for the bend in our setup. Our results are therefore in approximate agreement with ref. 43. The difference in path length in the $x z$ plane between the inner and outer wire surface (see Fig. 2) along the $90^{\circ}$ bend is $2.4 \mathrm{~mm}$. This corresponds to 8 ps time lag of waves travelling along the outside of the bend with respect to the inside of the bend, in agreement with the observed dispersion of the SPP.

These first experiments demonstrate that intense SPPs can be excited by grazing incidence CTR, generated by ultrashort relativistic electron bunches at the conical tip of a bare metal wire. The strong fields allow quantitative analysis and comparison to the theoretical predictions made in ref. 34. The SPPs were shown to propagate around a relatively tight bend which demonstrates the potential to use these waves for $\mathrm{THz}$ plasmonics and the possibility to develop endoscopic $\mathrm{THz}$ systems. Using state-of-the-art table-top-sized accelerators ${ }^{35}$, extremely intense $\mathrm{THz}$ pulses can be created by concentrating the full $\mathrm{THz}$ pulse on a small tip. A particularly exciting prospect is to use the nanocoulomb, sub-100-fs, GeV electron bunches that currently drive X-FELs ${ }^{25}$ to excite wire-based THz SPPs. Extrapolating our measured field value using the scaling of Equation (1), such bunches should generate $0.4 \mathrm{GV} \mathrm{m}^{-1} \mathrm{THz}$ SPPs on a millimetrediameter wire. By tapering this wire into a sharp point ${ }^{12-20}$, these $\mathrm{THz}$ pulses could be delivered in a micron-sized spot, resulting in unprecedented $\mathrm{THz}$ field strengths of $100 \mathrm{GV} \mathrm{m}^{-1}$ and beyond.

\section{Methods}

SPP formation by transition radiation. By firing electron bunches onto the tip of a tapered metal wire, the radially polarized field of the electron bunches is transferred to the wire and continues to propagate along the wire as an SPP. The physical mechanism behind this process can be elucidated using the field line model for transition radiation as described by Purcell ${ }^{36}$. Consider an electron travelling with velocity $v$ in the $z$ direction, reaching the tip of a metal wire with small cone angle at time $t=0$. This situation is sketched in Fig. 1 . For $t<0$, the field is that of an undisturbed charge travelling in vacuum. The thin metal wire has negligible influence because the field of the electron at relativistic speeds is confined to a thin disk perpendicular to the direction of motion. For $t>0$ when the electron is inside the metal wire, the electric fields are screened for an observer outside the metal. However, the screening is not instantaneous; instead it occurs on a sphere travelling outwards with the speed of light. Outside the sphere, indicated as region II in Fig. 1, the electric field is still that of the undisturbed charge in motion. Within the sphere, indicated as region I in Fig. 1, there is no electric field. However, the electric field lines in region II cannot abruptly end at the sphere; instead they follow the surface of the sphere ending on the metal wire where they induce a surface charge. This combination of electric fields and co-propagating surface charge is an SPP. As the electric field of an electron is very well matched to the cylindrically symmetric mode of a wire, it will excite a strong SPP. In case of an electron bunch rather than a single electron, the shell of field lines has a thickness $v \tau$, where $\tau$ is the time it takes the bunch to pass the metal surface. We can easily calculate the electric field on the metal wire at the location of the expanding shell using Gauss's law on the closed surface indicated by the red dashed line in the figure. Because this Gauss volume does not enclose any charge, the net flux through the surface is zero. Therefore, the flux contained in the expanding sphere equals the flux crossing the Gauss volume near the surface of the wire, both being equal to $q / \varepsilon_{0}$, with $q$ the bunch charge. On the surface of the wire, this flux is spread over an annulus with radius $R$ and width $c \tau$. This yields the field strength given by Equation (1).

Experimental setup. The experimental setup contains a 1.5 cell, $3 \mathrm{GHz}$ radio frequency accelerator operating at a repetition frequency of $3 \mathrm{~Hz}$. A Ti:Sapphire laser system produces pulses of $35 \mu \mathrm{J}$ at $267 \mathrm{~nm}$ wavelength (generated by frequency tripling of the Ti:Sapphire fundamental wavelength at $800 \mathrm{~nm}$ ) with a duration of approximately $120 \mathrm{fs}$ and a (RMS) radius of $1.5 \mathrm{~mm}$. These pulses hit the copper cathode of the accelerator and generate bunches with a charge of $160 \mathrm{pC}$. The electrons are accelerated to $3.1 \mathrm{MeV}$ and focussed by a quadrupole triplet onto the tip of the aluminium wire. The laser spot on the cathode is slightly elliptical, with an RMS radius of $0.1 \mathrm{~mm}$ in the horizontal direction and $0.15 \mathrm{~mm}$ in the vertical direction. At the position of the wire tip, the electron bunch is nearly circular with an RMS radius of $0.2 \mathrm{~mm}$. The RMS-bunch duration at the tip of the wire, calculated by particle tracking simulations (General Particle Tracer ${ }^{42}$ ) is $2.7 \mathrm{ps}$. The wire has a radius of $0.75 \mathrm{~mm}$ and a conical tip with a cone angle $2 \delta=8^{\circ}$. The distance between the cathode surface and the tip of the wire is $1.5 \mathrm{~m}$. Synchronization between the accelerator and the Ti:Sapphire laser system is better than $100 \mathrm{fs}$, using an AccTec phase-locked loop synchronizer ${ }^{37}$. A small part of the $800 \mathrm{~nm}$ laser energy $(\sim 1 \mu \mathrm{J})$ is split off before the third harmonic generation to be used as probe pulses for the electro-optic detection of the $\mathrm{THz}$ electric field strength $E_{y}$ in the ZnTe crystals. These pulses pass through a linear polarizer and a $\lambda / 4$ waveplate to make them circularly polarized. The [001] crystallographic axis of the $\mathrm{ZnTe}\langle 110\rangle$ crystal is aligned with the (horizontal) $x$ axis of the setup, perpendicular to the wire. In this configuration, the electro-optic phase shift, $\phi_{\mathrm{EO}}$ between the horizontal and vertical polarization directions is given by $\phi_{\mathrm{EO}}=n_{0}^{3} r_{41} k L E_{y}$, with $E_{y}$ the electric field strength in the $y$ direction, $n_{0}$ the refractive index of $\mathrm{ZnTe}$ at $800 \mathrm{~nm}, r_{41}$ the electro-optic coefficient of $\mathrm{ZnTe}$ involved in the Pockels effect, $k$ the wavenumber at $800 \mathrm{~nm}$ and $L$ the thickness of the crystal. A Wollaston prism is used to separate the horizontal and vertical components of the polarization of laser pulses. The first ZnTe crystal (the one positioned before the bent section (2) of the wire, see Fig. 2) is imaged through the Wollaston prism onto two charge-coupled device cameras. The pulses passing through the second ZnTe crystal (after the bend (2) in the wire) are focussed onto two photodiodes, after passing through the prism. In both cases, the relative difference of the intensity of the signal on the two detectors is proportional to the electric field in the $y$ direction: $\left(I_{1}-I_{2}\right) /\left(I_{1}+I_{2}\right)=\sin \left(\phi_{\mathrm{EO}}\right) \cong n_{0}^{3} r_{41} k L E_{y}$, for $\phi_{\mathrm{EO}} \ll 1$, with $I_{1,2}$ the intensity on each of the detectors. The thickness of both crystals is $L=0.5 \mathrm{~mm}$. For the calculation of the absolute value of the electric field strength $E_{y}$, we have used $n_{0}=2.85$ and $r_{41}=4.3 \mathrm{pm} \mathrm{V}^{-1}$ (ref. 38).

Data availability. The data that support the findings of this study are available from the corresponding author upon request.

\section{References}

1. Auston, D. H. \& Nuss, M. C. Electrooptic generation and detection of femtosecond electrical transients. IEEE J. Quantum Electron. 24, 184-197 (1988).

2. Yeh, K.-L., Hoffmann, M. C., Hebling, J. \& Nelson, K. A. Generation of $10 \mu$ ultrashort terahertz pulses by optical rectification. Appl. Phys. Lett. 90, 171121 (2007).

3. Bartel, T., Gaal, P., Reimann, K., Woerner, M. \& Elsaesser, T. Generation of single-cycle $\mathrm{THz}$ transients with high electric-field amplitudes. Opt. Lett. 30, 2805-2807 (2005).

4. Sell, A., Leitenstorfer, A. \& Huber, R. Phase-locked generation and fieldresolved detection of widely tunable terahertz pulses with amplitudes exceeding $100 \mathrm{MV}$ per cm. Opt. Lett. 33, 2767-2769 (2008).

5. Reimann, K. Terahertz radiation: a table-top source of strong pulses. Nat. Photon. 2, 596-597 (2008).

6. Mittleman, D. M. Frontiers in terahertz sources and plasmonics. Nat. Photon 7, 666-669 (2013).

7. Andrews, S. R. Microstructured terahertz waveguides. J. Phys. D Appl. Phys. 47, 374004 (2014).

8. Wang, K. \& Mittleman, D. M. Metal wires for terahertz wave guiding. Nature 432, 376-379 (2004)

9. Kampfrath, T., Tanaka, K. \& Nelson, K. A. Resonant and nonresonant control over matter and light by intense terahertz transients. Nat. Photon. 7, 680-690 (2013).

10. Yu, C., Fan, S., Sun, Y. \& Pickwel-MacPherson, E. The potential of terahertz imaging for cancer diagnosis: a review of investigations to date. Quant. Imaging Med. Surg. 2, 33-45 (2012).

11. Wallace, V. P. et al. Terahertz pulsed imaging of basal cell carcinoma ex vivo and in vivo. Br. J. Dermatol. 151, 424-432 (2004).

12. Maier, S. A., Andrews, S. R., Martin-Moreno, L. \& Garcia-Vidal, F. J. Terahertz surface plasmon-polariton propagation and focusing on periodically corrugated metal wires. Phys. Rev. Lett. 97, 176805 (2006).

13. Awad, M., Nagel, M. \& Kurz, H. Tapered Sommerfeld wire terahertz near-field imaging. Appl. Phys. Lett. 94, 051107 (2009).

14. Astley, V., Mendis, R. \& Mittleman, D. M. Characterization of terahertz field confinement at the end of a tapered metal wire waveguide. Appl. Phys. Lett. 95, 031104 (2009).

15. Wächter, M., Nagel, M. \& Kurz, H. Tapered photoconductive terahertz field probe tip with subwavelength spatial resolution. Appl. Phys. Lett. 95, 041112 (2009).

16. Liang, H., Ruan, S. \& Zhang, M. Terahertz surface wave propagation and focusing on conical metal wires. Opt. Express 16, 18241-18248 (2008).

17. Huber, A. J., Keilmann, F., Wittborn, J., Aizpurua, J. \& Hillenbrand, R. Terahertz near-field nanoscopy of mobile carriers in single semiconductor nanodevices. Nano Lett. 8, 3766-3770 (2008).

18. Schnell, M. et al. Nanofocusing of mid-infrared energy with tapered transmission lines. Nat. Photon. 5, 283-287 (2011).

19. Gramotnev, D. K. \& Bozhevolnyi, S. I. Plasmonics beyond the diffraction limit Nat. Photon. 4, 83-91 (2010).

20. Schuller, J. A. et al. Plasmonics for extreme light concentration and manipulation. Nat. Mater. 9, 193-204 (2010).

21. Huang, W. R. et al. Towards a THz-driven electron gun. Sci. Rep. 5, 14899 (2015). 
22. Eisele, M. et al. Ultrafast multi-terahertz nano-spectrometry with sub-cycle temporal resolution. Nat. Photon. 8, 841-845 (2014).

23. Seo, M. A. et al. Terahertz field enhancement by a metallic nano slit operating beyond the skin-depth limit. Nat. Photon. 3, 152-156 (2009).

24. Carr, G. L. et al. High-power terahertz radiation from relativistic electrons. Nature 420, 153-156 (2002).

25. Wu, Z. et al. Intense terahertz pulses from SLAC electron beams using coherent transition radiation. Rev. Sci. Instrum. 84, 022701 (2013).

26. van Tilborg, J. et al. Temporal characterization of femtosecond laser-plasmaaccelerated electron bunches using terahertz radiation. Phys. Rev. Lett. 96, 014801 (2006).

27. Jeon, T.-I., Zhang, J. \& Grischkowsky, D. THz Sommerfeld wave propagation on a single metal wire. Appl. Phys. Lett. 86, 161904 (2005).

28. Deibel, J. A., Wang, K., Escarra, M. D. \& Mittleman, D. M. Enhanced coupling of terahertz radiation to cylindrical wire waveguides. Opt. Express 14, 270-290 (2006).

29. Zheng, Z., Kanda, N., Konishi, K. \& Kuwata-Gonokami, M. Efficient coupling of broadband terahertz radial beams to metal wires. Opt. Express 21, 10642-10650 (2013).

30. Zhu, W., Agrawal, A., Cao, H. \& Nahata, A. Generation of broadband radially polarized terahertz radiation directly on a cylindrical metal wire. Opt. Express 16, 8433-8439 (2008).

31. Mittleman, D. M. Frontiers in terahertz sources and plasmonics. Nat. Photon. 7, 666-669 (2013).

32. Edelmann, A., Moeller, L. \& Jahns, J. Coupling of terahertz radiation to metallic wire using end-fire technique. Electron Lett. 49, 884-886 (2013).

33. Gong, S. et al. Electron beam excitation of surface plasmon polaritons. Opt. Express 22, 19252-19261 (2014).

34. Smorenburg, P. W., Op 't Root, W. P. E. M. \& Luiten, O. J. Direct generation of terahertz surface plasmon polaritons on a wire using electron bunches. Phys. Rev. B 78, 115415 (2008).

35. van der Geer, S. B. et al. Longitudinal phase-space manipulation of ellipsoidal electron bunches in realistic fields. Phys. Rev. ST Accel. Beams 9, 044203 (2006).

36. Purcell, E. M. Electricity and Magnetism (Cambridge University Press, 2011).

37. Brussaard, G. J. H. et al. Direct measurement of synchronization between femtosecond laser pulses and a $3 \mathrm{GHz}$ radio frequency electric field inside a resonant cavity. Appl. Phys. Lett. 103, 141105 (2013).

38. Sliker, T. R. \& Jost, J. M. Linear electro-optic effect and refractive indices of cubic ZnTe. J. Opt. Soc. Am. 56, 130-131 (1966).

39. Chen, Q., Tani, M., Jiang, Z. \& Zhang, X.-C. Electro-optic transceivers for terahertz-wave applications. J. Opt. Soc. Am. B 18, 823-831 (2001).

40. Planken, P. C. M., Nienhuys, H.-K., Bakker, H. J. \& Wenckebach, T. Measurement and calculation of the orientation dependence of terahertz pulse detection in ZnTe. J. Opt. Soc. Am. B 18, 313-317 (2001)

41. Goubau, G. Surface waves and their application to transmission lines. J. Appl. Phys. 21, 1119-1128 (1950).
42. Pöplau, G., van Rienen, U., van der Geer, B. \& de Loos, M. Multigrid algorithms for the fast calculation of space-charge effects in accelerator design. IEEE Trans. Magn. 40, 714-717 (2004).

43. Astley, V., Scheiman, J., Mendis, R. \& Mittleman, D. M. Bending and coupling losses in terahertz wire waveguides. Opt. Lett. 35, 553-555 (2010).

\section{Acknowledgements}

We thank the technical staff: Eddy Rietman, Harry van Doorn, and Ad Kemper, who were pivotal in constructing the experiment. We are also indebted to Koen Pieterse and his team of the TU/e ICMS Animation Studio for a beautiful and very instructive movie of the experiment. This work is part of the research programme of the Foundation for Fundamental Research on Matter (FOM), which is part of the Netherlands Organisation for Scientific Research (NWO)

\section{Author contributions}

O.J.L. conceived the experiments; W.P.E.M.R. performed the experiments; all the authors developed the theoretical background; W.P.E.M.R., G.J.H.B. and O.J.L. analysed the data all the authors co-wrote the paper.

\section{Additional information}

Supplementary Information accompanies this paper at http://www.nature.com/ naturecommunications

Competing financial interests: The authors declare no competing financial interests.

Reprints and permission information is available online at http://npg.nature.com/ reprintsandpermissions/

How to cite this article: op 't Root, W. P. E. M. et al. Single-cycle surface plasmon polaritons on a bare metal wire excited by relativistic electrons. Nat. Commun. 7, 13769 doi: $10.1038 /$ ncomms13769 (2016)

Publisher's note: Springer Nature remains neutral with regard to jurisdictional claims in published maps and institutional affiliations.

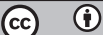

This work is licensed under a Creative Commons Attribution 4.0 International License. The images or other third party material in this article are included in the article's Creative Commons license, unless indicated otherwise in the credit line; if the material is not included under the Creative Commons license, users will need to obtain permission from the license holder to reproduce the material. To view a copy of this license, visit http://creativecommons.org/licenses/by/4.0/

(C) The Author(s) 2016 